\title{
Subcuticular sutures reduce surgical site infection after repeat liver resection: a matched cohort analysis
}

\author{
Shintaro Yamazaki, Tadatoshi Takayama*, Yoritaka Matsuno, Yusuke Mitsuka, Nao Yoshida, \\ Masamichi Moriguchi, Tokio Higaki
}

Department of Digestive Surgery, Nihon University School of Medicine, 30-1 Ohyaguchikami-machi, Itabashi-ku, Tokyo 173-8610, Japan

SUMMARY Liver cancer frequently requires repeated liver resections due to the high recurrence rate. The aim of this study was to clarify whether subcuticular sutures reduce wound complication rates following repeat incisions. Data from 382 repeated liver resections in 1,245 consecutive patients were assessed. Patients were divided into a Subcuticular sutures group and a Skin staples group on the basis of the wound-closure method. To avoid bias in analysing wound complications, data were matched to adjust for patient background and operation variables. After matching, 82 matched, paired patients with subcuticular sutures or skin staples were compared. Total wound complication rate was significantly lower with subcuticular sutures than with skin staples $(8.5 \%$ vs. $20.7 \%, p=$ $0.027)$. Incisional surgical site infection was also lower with subcuticular sutures than with skin staples $(6.1 \%$ vs. $17.1, p=0.028)$. Univariate analysis revealed 4 factors associated with wound complications: body mass index; serum albumin concentration; wound length; and closure with skin staples. Multivariate analysis revealed closure with skin staples (odds ratio, 2.91; 95\% confidence interval, 1.07-7.94; $p=0.037$ ) as the only independent factor negatively associated with wound complications. Subcuticular sutures appear to reduce wound complications compared to skin staples following repeat incision for liver resection.

Keywords surgical site infection, subcuticular suture, repeat liver resection

\section{Introduction}

Using subcuticular sutures in wound closure reportedly contributes to reducing wound complication rates $(1,2)$. However, subcuticular sutures have shown superiority only in sub-group analyses of clean-contaminated surgeries, such as digestive surgeries $(3,4)$.

In hepatobiliary surgery, the wound complication rate is still over $10 \%$ and is considered to contribute to prolonged hospitalization (5-7). Furthermore, liver cancers such as hepatocellular carcinoma and colorectal metastasis show a high frequency of recurrence and around $30-50 \%$ of patients require repeat laparotomy $(8,9)$. Re-laparotomy using the same incision is thus frequent, and has been considered to be negatively associated with wound complications. Attempts to minimize wound complications have included intraoperative administration of antimicrobials, irrigation of wounds before closure, use of absorbable sutures in the subcutaneous layer and application of woundprotective dressing materials. However, none of these steps have proven particularly effective (10-12).

No studies have focused on factors associated with wound complications following repeated incisions for liver resection. This may be because the development of wound complications is a multifactorial issue, involving factors such as operation scale, procedures, background of chronic liver diseases, longer operation times and greater length of the surgical incision for thoracotomy. We therefore analysed factors associated with wound complications after adjusting for perioperative variables to minimize selection bias.

\section{Materials and Methods}

\subsection{Study design}

Between April 2001 and December 2015, data were collected from 1,245 consecutive patients who underwent hepatic resection for liver cancer. The target for analysis was repeated laparotomy using the same incision in patients undergoing skin closure with either subcuticular sutures or metallic skin staples, without use of subcutaneous drainage tubes. Subcutaneous drainage tubes were avoided because we have previously applied this method to minimize wound complications (7). 
Also, the patients who have large incisional hernia caused by previous operation was excluded from this study. Patients were divided into groups according to the use of subcuticular sutures or skin staples for wound closure.

\subsection{Wound closure}

A J-shaped incision was the most common incision, with thoracotomy added only when the tumor was located in the posterior segment or caudate lobe. At wound closure, surgical gloves and instruments were changed and the subcutaneous space was irrigated with saline. Routine approximation of the fat layer with 3-0 multifilament absorbable suture (polyglycolic acid, OPEPOLYX $^{\circledR}$ N; Alfresa Pharma Corporation, Tokyo, Japan) was performed before skin closure. Finally, 3-0 monofilament absorbable sutures (polydioxanone, MONODIOX $^{\circledR}$; Alfresa Pharma Corporation) were used for subcuticular sutures with both interval and length of bite of sutures set at $15 \mathrm{~mm}$. In the skin staples group, metallic skin staples (Appose ULC 35W; Medtronic, Tokyo, Japan) $10 \mathrm{~mm}$ in size were used. Peripheral blood circulation in the scar tissue was poor and may thus create poor conditions for tissue adaption. To remove the tissue of poor local skin blood circulation, the scar tissue was routinely removed at incision in case of repeat laparotomy.

\subsection{Assessment}

The total incidence of all wound complications was compared between the Subcuticular and Skin staples groups before and after matching. Finally, factors associated with wound complications were analysed and whether subcuticular sutures were superior to skin staples in repeated incision was assessed.

\subsection{Definitions}

According to the Centers for Disease Control and Prevention guideline (13), superficial incisional surgical site infection (SSI) was defined as an infection occurring within 30 days of surgery and arising only in the skin or subcutaneous tissue. Deep incisional SSI was defined as infection occurring within 30 days of surgery and arising only in the fascial or muscle layers. Wound complications were defined as the presence of signs relating to treatment: wound disruption, stitch abscess, abscess, seroma or hematoma, or superficial or deep incisional SSI. All information on wound complications was collected on a uniform sheet in our unit.

\subsection{Data manipulation for matching}

First, raw preoperative data for patient characteristics, tumor status and operation-related variables were compared between groups to assess wound complications. Next, biases in background data were examined, then data were matched using Greedy matching methods (14) to the data of nearest-neighbor patients. Written informed consent for clinical analysis was obtained from each patient undergoing liver resection. All analyses were performed in accordance with the ethics guidelines for clinical studies at Nihon University Itabashi Hospital (RK-170214-03).

\subsection{Statistical analyses}

Data are expressed as medians and ranges or as absolute values and percentages. Student's $t$-test, the $\chi 2$ test, and Fisher's exact test were used for univariate analyses, as appropriate. Continuous variables were compared using the Mann-Whitney $U$-test. Multivariate analysis was performed using logistic regression methods. Odds ratios with $95 \%$ confidence intervals derived from logistic regression analysis were calculated. Values of $P<0.05$ were considered to indicate statistical significance. All analyses were performed using JMP version 13.2 statistical software (SAS Institute, Cary, NC, USA).

\section{Results}

\subsection{Study flow}

Between April 2001 and December 2015, a total of 1,245 patients underwent hepatic resection for liver cancer at our institution (Figure 1). Among these, 863 patients were excluded from analysis because of primary operation for liver resection. Next, 116 patients were excluded because of wound closure methods that were neither subcuticular sutures nor metallic skin staples. Then, the remaining 266 patients were divided into two groups according to the method of wound closure. To eliminate the influence of background, matching was performed before analysis. Finally, 82 patients from each group were assessed and comparisons were made between subcuticular sutures (Subcuticular sutures group) and metallic skin staples (Skin staples group).

\subsection{Raw data analysis and matching}

Before matching, neither patient background characteristics nor liver functional reserve differed significantly between groups (Table 1). However, the rate of hepatitis $\mathrm{C}$ infection was significantly higher in the Skin staples group (31.5\%) than in the Subcuticular sutures group $(14.8 \%, p=0.004)$. As for operationrelated variables, median wound length was significantly longer in the Subcuticular sutures group $(36 \mathrm{~cm}$, range, $20-56 \mathrm{~cm})$ than in the Skin staples group $(31 \mathrm{~cm}, 22-48$ $\mathrm{cm} ; p=0.039$ ).

Operation-related variables such as wound length differed markedly between groups, and so were matched 


\section{Study flow}

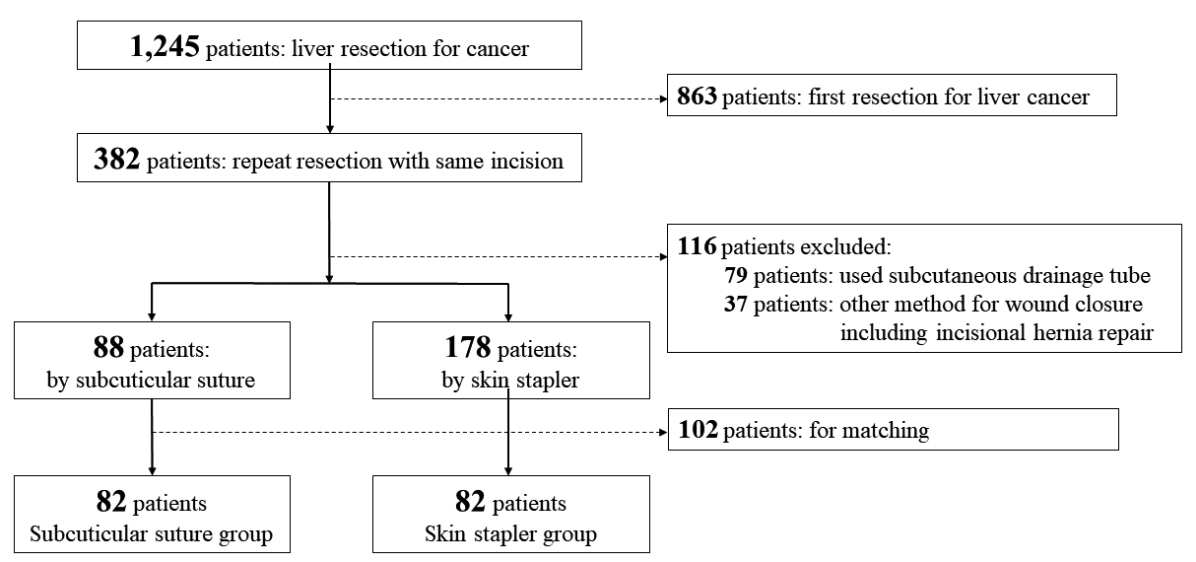

Figure 1. Flow diagram of the patients. A total of 1,245 patients underwent hepatic resection for liver cancer at our institution. 863 patients were excluded from analysis because of primary operation for liver resection. Finally, 82 patients from each group were assessed and comparisons were made between subcuticular sutures (Subcuticular sutures group) and metallic skin staples (Skin staples group).

Table 1. Raw patient's baseline characteristics

\begin{tabular}{|c|c|c|c|}
\hline & Subcuticular stures $(n=88)$ & Staples $(n=178)$ & $p$-value \\
\hline Age (year) & $68(44-79)$ & $69(26-82)$ & 0.671 \\
\hline Body Mass Index & $22.7(15.8-32.3)$ & $23.1(15.6-30.8)$ & 0.611 \\
\hline Diabetes mellitus & $27(30.7)$ & $60(33.7)$ & 0.621 \\
\hline Anticoagulation therapy & $13(14.8)$ & $34(19.1)$ & 0.384 \\
\hline Preoperative chemotherapy & $23(26.1)$ & $32(18.0)$ & 0.122 \\
\hline History of smorking & $13(14.8)$ & $33(18.5)$ & 0.445 \\
\hline ICG-R15 (\%) & $10.7(1.9-33.3)$ & $11.7(2.5-32.7)$ & 0.411 \\
\hline \multicolumn{4}{|l|}{ Viral infection } \\
\hline HBV & $20(22.7)$ & $30(16.9)$ & 0.249 \\
\hline $\mathrm{HCV}$ & $13(14.8)$ & $56(31.5)$ & 0.004 \\
\hline Total-bilirubin (mg/dL) & $0.73(0.20-1.59)$ & $0.59(0.25-1.77)$ & 0.748 \\
\hline Prothrombin time (\%) & $100(80-100)$ & $100(74-100)$ & 0.299 \\
\hline Albumin $(\mathrm{g} / \mathrm{dL})$ & $4.3(2.5-5.2)$ & $4.2(3.1-5.7)$ & 0.584 \\
\hline Child-pugh class A & $84(95.5)$ & $174(97.8)$ & 0.302 \\
\hline Bile leakage & $8(9.8)$ & $8(9.8)$ & 1.000 \\
\hline $\mathrm{HbA1c}(\%)$ & $5.9(4.6-8.8)$ & $5.8(4.1-8.8)$ & 0.831 \\
\hline Platelet count $\left(10^{4} \mu \mathrm{L}\right)$ & $17.4(7.8-45.8)$ & $17.8(6.3-44.1)$ & 0.671 \\
\hline Creatinine $(\mathrm{mg} / \mathrm{dL})$ & $0.88(0.36-1.49)$ & $0.76(0.48-1.44)$ & 0.142 \\
\hline \multicolumn{4}{|l|}{ Diseases } \\
\hline Hepatocellular carcinoma & $47(53.4)$ & $105(59.0)$ & 0.387 \\
\hline \multicolumn{4}{|l|}{ Operation related variables } \\
\hline Segmentectomy & $9(10.2)$ & $20(11.2)$ & 0.804 \\
\hline Lobectomy or extented lobectomy & $8(9.1)$ & $13(7.3)$ & 1.000 \\
\hline Operation time (min) & $341(165-681)$ & $361(126-812)$ & 0.698 \\
\hline Blood loss (mL) & $249(2-1114)$ & $261(10-945)$ & 0.682 \\
\hline Wound length $(\mathrm{cm})$ & $36(20-56)$ & $31(22-48)$ & 0.039 \\
\hline Wound thickness (mm) & $21(6-37)$ & $22(6-47)$ & 0.516 \\
\hline Bile leakage & $8(9.8)$ & $18(10.1)$ & 0.792 \\
\hline With thoracotomy & $37(42.1)$ & $84(47.2)$ & 0.427 \\
\hline Clavien-Dindo classification grade ( $\geq$ IIIb) & $3(3.4 \%)$ & $5(2.8 \%)$ & 0.746 \\
\hline
\end{tabular}

ICG-R15, indocyanine green retention rate at 15 minutes; HBV, Hepatitis B virus; HCV, Hepatitis C virus.

for to avoid bias (Table 2). After matching, no significant differences in background status and operation-related variables of patients were identified.

\subsection{Wound complications}

In matched groups, the total complication rate was significantly lower in the Subcuticular sutures group $(8.5 \%)$ than in the Skin staples group $(20.7 \%, p=$ 0.027 ) (Table 3). Incisional SSI was significantly less frequent in the Subcuticular sutures group (6.1\%) than in the Skin staples group $(17.1 \%, p=0.028)$. Rates of other wound complications did not differ significantly between groups. 
Table 2. Baseline characteristics after matching

\begin{tabular}{|c|c|c|c|}
\hline & Subcuticular stures $(n=82)$ & Staples $(n=82)$ & $p$-value \\
\hline Age (year) & $68(44-79)$ & $69(26-80)$ & 0.683 \\
\hline Body Mass Index & $22.6(16.2-32.3)$ & $22.3(15.6-30.8)$ & 0.545 \\
\hline Diabetes mellitus & $27(32.9)$ & 27 (32.9) & 1.000 \\
\hline Anticoagulation therapy & $12(14.6)$ & $13(15.9)$ & 0.828 \\
\hline Preoperative chemotherapy & $20(24.4)$ & $14(17.1)$ & 0.248 \\
\hline History of smorking & $13(14.8)$ & $16(19.5)$ & 0.539 \\
\hline ICG-R15 (\%) & $10.8(1.9-32.8)$ & $11.6(2.5-32.5)$ & 0.240 \\
\hline \multicolumn{4}{|l|}{ Viral infection } \\
\hline HBV & $18(22.0)$ & $9(11.0)$ & 0.058 \\
\hline $\mathrm{HCV}$ & $12(14.6)$ & $19(23.2)$ & 0.163 \\
\hline Total-bilirubin (mg/dL) & $0.60(0.20-1.69)$ & $0.60(0.25-1.65)$ & 0.772 \\
\hline Prothrombin time (\%) & $100(80-100)$ & $100(74-100)$ & 0.219 \\
\hline Albumin (g/dL) & $4.3(2.5-5.1)$ & $4.2(3-5.7)$ & 0.594 \\
\hline Child-pugh class A & $80(97.6)$ & $78(95.1)$ & 0.406 \\
\hline $\mathrm{HbA} 1 \mathrm{c}(\%)$ & $5.9(4.6-8.8)$ & $5.9(4.8-8.0)$ & 0.934 \\
\hline Platelet count $\left(10^{4} \mu \mathrm{L}\right)$ & $17.5(7.8-45.8)$ & $18.6(6.3-39.1)$ & 0.668 \\
\hline Creatinine (mg/dL) & $0.81(0.37-1.45)$ & $0.79(0.48-1.44)$ & 0.066 \\
\hline \multicolumn{4}{|l|}{ Diseases } \\
\hline Hepatocellular carcinoma & $39(47.6)$ & $43(52.4)$ & 0.520 \\
\hline \multicolumn{4}{|l|}{ Operation related variables } \\
\hline Segmentectomy & $9(11.0)$ & $12(14.6)$ & 0.483 \\
\hline Lobectomy or extented lobectomy & $5(6.1)$ & $5(6.1)$ & 1.000 \\
\hline Operation time $(\mathrm{min})$ & $339(165-656)$ & $329(134-805)$ & 0.785 \\
\hline Blood loss $(\mathrm{mL})$ & $242(2-1114)$ & $238(10-945)$ & 0.631 \\
\hline Wound length $(\mathrm{cm})$ & $35(20-51)$ & $34(23-47)$ & 0.488 \\
\hline Wound thickness (mm) & $21(6-37)$ & $21(7-46)$ & 0.693 \\
\hline Bile leakage & $8(9.8)$ & $8(9.8)$ & 1.000 \\
\hline With thoracotomy & $36(43.9)$ & $38(46.3)$ & 0.754 \\
\hline Clavien-Dindo classification grade $(\geq \mathrm{IIIb})$ & $3(3.7 \%)$ & $3(3.7 \%)$ & 1.000 \\
\hline
\end{tabular}

ICG-R15, indocyanine green retention rate at 15 minutes; HBV, Hepatitis B virus; HCV, Hepatitis C virus.

Table 3. Complications

\begin{tabular}{|c|c|c|c|c|}
\hline & Subcuticular sutures $(n=82)$ & Staples $(n=82)$ & Odds ratio $(95 \% \mathrm{CI})$ & $p$-value \\
\hline Total wound complication rate & $7(8.5 \%)$ & $17(20.7 \%)$ & $2.80(1.09-7.18)$ & 0.027 \\
\hline Incisional SSI & $5(6.1 \%)$ & $14(17.1 \%)$ & $3.17(1.08-9.26)$ & 0.028 \\
\hline Organ space SSI & $1(1.2 \%)$ & $2(2.4 \%)$ & $2.03(0.18-22.78)$ & 0.560 \\
\hline Wound separation & $0(0 \%)$ & $0(0 \%)$ & 0 & 1.000 \\
\hline Heaematoma & $1(1.2 \%)$ & $1(1.2 \%)$ & $1.00(0.06-16.26)$ & 1.000 \\
\hline Seroma & $1(1.2 \%)$ & $2(2.4 \%)$ & $2.03(0.18-22.78)$ & 0.560 \\
\hline
\end{tabular}

SSI, surgical site infection.

\subsection{Risk analysis}

In univariate analysis, body mass index (BMI) ( $p=$ $0.033)$, serum albumin value $(p=0.031)$, wound length ( $p$ $=0.023)$ and closure with skin staples $(p=0.027)$ were the factors predicting wound complications (Table 4). In multivariate analysis, the only factor showing a negative independent association with wound complications was closure with skin staples (odds ratio, 2.91; 95\% confidence interval, [1.07-7.94], $p=0.037$ ).

\section{Discussion}

Significant differences in complication rates were observed with subcuticular sutures compared to skin staples, especially for incisional SSI. This study thus suggested the superiority of subcuticular sutures as a standard procedure in repeated incisions for liver resection. To the best of our knowledge, this study is the first to target repeated incisions, which has been considered a key risk factor for wound complications. 24 We have therefore conducted a study focusing on repeated liver resection as a potential risk factor for the development of wound complications.

Wound complication rates remain high, within the range of $6-33 \%$, and have yet to show any substantial reductions in open liver surgery over the last decade $(5-7,10,15)$. Even though wound complications were not severe and did not affect patient survival, such complications cause discomfort in patients, prolong 
Table 4. Logistic regression analysis for the risk of wound complication

\begin{tabular}{|c|c|c|c|c|c|c|}
\hline \multirow{2}{*}{ Risk factor } & \multicolumn{3}{|c|}{ Univariate analysis } & \multicolumn{3}{|c|}{ Multivariate analysis } \\
\hline & odds ratio & $95 \% \mathrm{CI}$ & $p$-value & odds ratio & $95 \% \mathrm{CI}$ & $p$-value \\
\hline \multicolumn{7}{|l|}{ Patient's related variables } \\
\hline Age $(\geq 75$ years-old $)$ & 1.34 & $0.45-3.95$ & 0.596 & 1.94 & $0.57-6.63$ & 0.290 \\
\hline Gender (male) & 2.51 & $0.71-8.92$ & 0.142 & & & \\
\hline Body Mass Index $(\geq 25)$ & 2.62 & $1.06-6.48$ & 0.033 & & & \\
\hline Wound closure (Staples) & 2.80 & $1.09-7.18$ & 0.027 & 2.91 & $1.07-7.94$ & 0.037 \\
\hline Albumin $(<3.5 \mathrm{~g} / \mathrm{dL})$ & 4.86 & $1.01-23.25$ & 0.031 & 4.36 & $0.74-25.58$ & 0.102 \\
\hline Child-pugh class A & 3.09 & $0.53-17.90$ & 0.187 & & & \\
\hline Creatinine $(\geq 1.00 \mathrm{mg} / \mathrm{dL})$ & 0.97 & $0.26-3.58$ & 0.961 & & & \\
\hline Presence of diabetes mellitus & 1.27 & $0.52-3.11$ & 0.606 & & & \\
\hline ICG-R15 ( $\geq 15 \%)$ & 2.41 & $0.98-5.94$ & 0.051 & & & \\
\hline Preoperative chemotherapy & 1.01 & $0.35-2.93$ & 0.989 & & & \\
\hline \multicolumn{7}{|l|}{ Operation related variables } \\
\hline Lobectomy or extented lobectomy & 1.50 & $0.30-7.53$ & 0.620 & 0.97 & $0.16-5.66$ & 0.969 \\
\hline Operation time $(\geq 300 \mathrm{~min})$ & 2.46 & $0.87-6.97$ & 0.083 & 2.44 & $0.64-9.34$ & 0.193 \\
\hline Blood loss $(\geq 300 \mathrm{~mL})$ & 2.13 & $0.89-5.10$ & 0.086 & 1.67 & $0.64-4.38$ & 0.299 \\
\hline Subcutaneous drainage & 1.15 & $0.44-2.98$ & 0.780 & & & \\
\hline With thoracotomy & 1.87 & $0.78-4.49$ & 0.159 & & & \\
\hline Wound length $(\geq 35 \mathrm{~cm})$ & 2.88 & $1.13-7.39$ & 0.023 & 2.08 & $0.67-6.43$ & 0.202 \\
\hline Wound thickness $(\geq 30 \mathrm{~mm})$ & 1.55 & $0.47-5.11$ & 0.469 & 0.92 & $0.24-3.47$ & 0.902 \\
\hline Bille leakage & 0.82 & $0.17-3.85$ & 0.799 & & & \\
\hline
\end{tabular}

SSI, surgical site infection.

hospitalization and increase the total medical cost (7). Regarding SSI in the abdominal space, we have successfully reduced abdominal space infection based on results from two clinical studies $(16,17)$. In contrast, a randomized controlled trial we performed to examine the effects of a subcutaneous drainage tube (UMIN000004437) on wound complications failed to reduce wound complications, and identified no specific factors related to wound complications (7). Because the development of wound complications is multifactorial, operation-related factors need to be considered along with systemic diseases such as diabetes merits, chronic hepatitis and metabolic disease. Moreover, nutritional status may affect wound adaptation in cases of liver cirrhosis. Using the matched cohort method, factors associated with wound complications were adjusted in the present study before analysis.

In multivariate analysis, use of skin staples was the only independent factor negatively associated with wound complications. In the present study, the total wound complication rate in the Subcuticular group was $8.5 \%$, appearing preferable to the $13.1 \%(34 / 260$ patients) in our previous study using a subcutaneous drainage tube (7). We speculated that peripheral blood circulation was poor for the scar tissue and may thus have created suboptimal conditions for wound adaption in repeated resection. In such situations, layer-to-layer fixation such as subcuticular sutures may help reduce wound complications (2-4).

In univariate analysis, BMI and wound length were predictors of wound complications. These results support minimally invasive hepatobiliary surgeries such as laparoscopic liver resection as one possible option to reduce wound complication rates. Indeed, the rate of severe complications was similar between open and laparoscopic surgeries, while the total wound complication rate was lower and operation time was shorter with laparoscopic surgery than with open surgery (18-20). Laparoscopic surgery is thus one feasible option when the tumour extension is preferable for laparoscopic surgery.

In our previous study, we focused on reducing the subcutaneous accumulation of effusions (7). However, we found no preventive effects of subcutaneous drain placement against wound infection. Because the effusion of subcutaneous tissue was smaller than we expected. Thus, we routinely performed subcutaneous suturing of the fat layer using absorbable sutures before skin closure for all patients to reduce dead space in subcutaneous tissue. Especially, the peripheral blood circulation in the repaired connective tissue may be interrupted by repeat incision and may thus create poor conditions for tissue adaption. Using with subcuticular sutures, the tightened tissue space may have contributed to reduced bacterial penetration into the subcutaneous tissue, especially with thick and long incisions.

Limitations of this study were that: $i$ ) randomization was not performed because of the retrospective nature of the study; and ii) laparoscopic surgeries were not included. Therefore, we are now initiating the largest $\mathrm{RCT}$ regarding wound closure in liver resection, to confirm the superiority of subcutaneous suture (UMIN000036670).

In conclusion, subcuticular sutures can be recommended as a standard procedure for wound closure following repeated incision in open liver surgery. 


\section{References}

1. Johnson RG, Cohn WE, Thurer RL, McCarthy JR, Sirois CA, Weintraub RM. Cutaneous closure after cardiac operations: a controlled, randomized, prospective comparison of subcuticular versus staple closures. Ann Surg. 1997; 226:606-612.

2. Basha SL, Rochon ML, Quiñones JN, Coassolo KM, Rust OA, Smulian JC. Randomized controlled trial of wound complication rates of subcuticular suture $v s$ staples for skin closure at cesarean delivery. Am J Obstet Gynecol. 2010 ; 203:285.e1-8.

3. Tsujinaka T, Yamamoto K, Fujita J, et al. Clinical Study Group of Osaka University on Section of Risk Management. Subcuticular sutures versus staples for skin closure after open gastrointestinal surgery: a phase 3 , multicentre, open-label, randomised controlled trial. Lancet. 2013; 382:1105-1112.

4. Kobayashi S, Ito M, Yamamoto S, Kinugasa Y, Kotake M, Saida Y, Kobatake T, Yamanaka T, Saito N, Moriya Y. Randomized clinical trial of skin closure by subcuticular suture or skin stapling after elective colorectal cancer surgery. Br J Surg. 2015; 102:495-500.

5. Kobayashi S, Gotohda N, Nakagohri T, Takahashi S, Konishi M, Kinoshita T. Risk factors of surgical site infection after hepatectomy for liver cancers. World $\mathrm{J}$ Surg. 2009; 33:312-317.

6. Moreno Elola-Olaso A, Davenport DL, Hundley JC, Daily MF, Gedaly R. Predictors of surgical site infection after liver resection: a multicentre analysis using National Surgical Quality Improvement Program data. HPB (Oxford). 2012; 14:136-41.

7. Nakayama H, Takayama T, Okubo T, Higaki T, Midorikawa Y, Moriguchi M, Aramaki O, Yamazaki S. Subcutaneous drainage to prevent wound infection in liver resection: a randomized controlled trial. J Hepatobiliary Pancreat Sci. 2014; 21:509-517.

8. Kishi Y, Shimada K, Nara S, Esaki M, Kosuge T. Role of hepatectomy for recurrent or initially unresectable hepatocellular carcinoma. World J Hepatol. 2014; 6:836843.

9. Andreou A, Brouquet A, Abdalla EK, Aloia TA, Curley SA, Vauthey JN. Repeat hepatectomy for recurrent colorectal liver metastases is associated with a high survival rate. HPB (Oxford). 2011; 13:774-782.

10. Tanaka K, Matsuo K, Kawaguchi D, Murakami T, Hiroshima Y, Hirano A. Sato S, Endo I, Taguri M Koda $\mathrm{K}$. Randomized clinical trial of peritoneal lavage for preventing surgical site infection in elective liver surgery. J Hepatobiliary Pancreat Sci. 2015; 22:446-53.

11. Mueller TC, Loos M, Haller B, Mihaljevic AL, Nitsche U,
Wilhelm D, Friess H, Kleeff J, Bader FG. Intra-operative wound irrigation to reduce surgical site infections after abdominal surgery: a systematic review and meta-analysis. Langenbecks Arch Surg. 2015; 400:167-181.

12. Mihaljevic AL, Schirren R, Özer M, et al. Multicenter double-blinded randomized controlled trial of standard abdominal wound edge protection with surgical dressings versus coverage with a sterile circular polyethylene drape for prevention of surgical site infections: a CHIR-Net trial (BaFO; NCT01181206). Ann Surg. 2014; 260:730-737.

13. Mangram AJ, Horan TC, Pearson ML, Jarvis WR. Guideline for prevention of surgical site infection, 1999. Hospital Infection Control Practices Advisory Committee. Infect Control Hosp Epidemiol, 1999; 20:250-278.

14. Austin PC. An introduction to propensity score methods forreducing the effects of confounding in observational studies. Multivariate Behav Res. 2011; 46:399-424.

15. Harimoto N, Shirabe K, Abe T, Yukaya T, Tsujita E, Gion T, Kajiyama K, Nagaie T. Prospective randomized controlled trial investigating the type of sutures used during hepatectomy. World J Gastroenterol. 2011; 17:2338-42.

16. Yamazaki S, Takayama T, Moriguchi M, Mitsuka Y, Okada S, Midorikawa Y, Nakayama H, Higaki T. Criteria for drain removal following liver resection. Br J Surg. 2012; 11:1584-90.

17. Mitsuka Y, Yamazaki S, Yoshida N, Masamichi M, Higaki T, Takayama T. Prospective Validation of Optimal Drain Management "The $3 \times 3$ Rule" after Liver Resection. World J Surg. 2016; 40:2213-2220.

18. Fretland Å, Dagenborg VJ, Bjørnelv GMW, et al. Laparoscopic Versus Open Resection for Colorectal Liver Metastases: The OSLO-COMET Randomized Controlled Trial. Ann Surg. 2018; 267:199-207.

19. Ratti F, Fiorentini G, Cipriani F, Catena M, Paganelli M, Aldrighetti L. Laparoscopic vs Open Surgery for Colorectal Liver Metastases. JAMA Surg. 2018; 153:1028-1035.

20. Yamazaki S, Takayama T. Current topics in liver surgery. Ann Gastroenterol Surg. 2019; 3:146-159.

Received August 8, 2020; Revised September 15, 2020; Accepted September 18, 2020.

*Address correspondence to:

Tadatoshi Takayama, Department of Digestive Surgery, Nihon University School of Medicine, 30-1 Ohyaguchikami-machi, Itabashi-ku, Tokyo 173-8610, Japan.

E-mail: takayama.tadatoshi@nihon-u.ac.jp

Released online in J-STAGE as advance publication September 30, 2020. 